\title{
Game Theory
}

\author{
Lecture Notes By
}

Y. Narahari

Department of Computer Science and Automation

Indian Institute of Science

Bangalore, India

July 2012

\section{The Revenue Equivalence Theorem}

Note: This is a only a draft version, so there could be flaws. If you find any errors, please do send email to hari@csa.iisc.ernet.in. A more thorough version would be available soon in this space.

\section{Revenue Equivalence Theorem}

In this section, we prove two results that show the revenue equivalence of certain classes of auctions. The first theorem is a general result that shows the revenue equivalence of two auctions that satisfy certain conditions. The second result is a more specific result that shows the revenue equivalence of four different types of auctions (English auction, Dutch auction, first price auction, and second price auction) in the special context of an auction of a single indivisible item. The proof of the second result crucially uses the first result.

\subsection{Revenue Equivalence of Two Auctions}

Assume that $y_{i}(\theta)$ is the probability of agent $i$ getting the object when the vector of announced types is $\theta=\left(\theta_{1}, \ldots, \theta_{n}\right)$. The expected payoff to the buyer $i$ with a type profile $\theta=\left(\theta_{1}, \cdots, \theta_{n}\right)$ will be $y_{i}(\theta) \theta_{i}+t_{i}(\theta)$. The set of allocations is given by

$$
K=\left\{\left(y_{1}, \cdots, y_{n}\right): y_{i} \in[0,1] \forall i=1, \cdots, n ; \sum_{i=1}^{n} y_{i} \leq 1\right\} .
$$

As earlier, let $\overline{y_{i}}\left(\hat{\theta}_{i}\right)=E_{\theta_{-i}}\left[y_{i}\left(\hat{\theta}_{i}, \theta_{-i}\right)\right]$ be the probability that agent $i$ gets the object conditional to announcing his type as $\hat{\theta}_{i}$, with the rest of the agents announcing their types truthfully. Similarly, $\overline{t_{i}}\left(\hat{\theta}_{i}\right)=E_{\theta_{-i}}\left[t_{i}\left(\hat{\theta}_{i}, \theta_{-i}\right)\right]$ denotes the expected payment received by agent $i$ conditional to announcing his type as $\hat{\theta}_{i}$, with the rest of the agents announcing their types truthfully. Let $\overline{v_{i}}\left(\hat{\theta}_{i}\right)=\bar{y}_{i}\left(\hat{\theta}_{i}\right)$. Then,

$$
U_{i}\left(\theta_{i}\right)=\overline{y_{i}}\left(\theta_{i}\right) \theta_{i}+\overline{t_{i}}\left(\theta_{i}\right)
$$

denotes the payoff to agent $i$ when all the buying agents announce their types truthfully. We now state and prove an important proposition. 
Theorem 1.1 Consider an auction scenario with:

1. $n$ risk-neutral bidders (buyers) $1,2, \cdots, n$

2. The valuation of bidder $i(i=1, \cdots, n)$ is a real interval $\left[\underline{\theta_{i}}, \overline{\theta_{i}}\right] \subset \mathbb{R}$ with $\underline{\theta_{i}}<\overline{\theta_{i}}$.

3. The valuation of bidder $i(i=1, \cdots, n)$ is drawn from $\left[\theta_{i}, \overline{\theta_{i}}\right]$ with a strictly positive density $\phi_{i}()>$.0 . Let $\Phi_{i}($.$) be the cumulative distribution function.$

4. The bidders' types are statistically independent.

Suppose that a given pair of Bayesian Nash equilibria of two different auction procedures are such that:

- For every bidder $i$, for each possible realization of $\left(\theta_{1}, \cdots, \theta_{n}\right)$, bidder $i$ has an identical probability of getting the good in the two auctions.

- Every bidder $i$ has the same expected payoff in the two auctions when his valuation for the object is at its lowest possible level.

Then the two auctions generate the same expected revenue to the seller.

Before proving the theorem, we elaborate on the first assumption above, namely risk neutrality. A bidder is said to be:

- risk-averse if his utility is a concave function of his wealth; that is, an increment in the wealth at a lower level of wealth leads to an increment in utility that is higher than the increase in utility due to an identical increment in wealth at a higher level of wealth;

- risk-loving if his utility is a convex function of his wealth; that is, an increment in the wealth at a lower level of wealth leads to an increment in utility that is lower than the increase in utility due to an identical increment in wealth at a higher level of wealth; and

- risk-neutral if his utility is a linear function of his wealth; that is, an increment in the wealth at a lower level of wealth leads to the same increment in the utility as an identical increment would yield at a higher level of wealth.

Proof: By the revelation principle, it is enough that we investigate two Bayesian incentive compatible social choice functions in this auction setting. It is enough that we show that two Bayesian incentive compatible social choice functions having (a) the same allocation functions $\left(y_{1}(\theta), \cdots, y_{n}(\theta)\right) \forall \theta \in \Theta$, and (b) the same values of $U_{1}\left(\theta_{1}\right), \cdots, U_{n}\left(\theta_{n}\right)$ will generate the same expected revenue to the seller.

We first derive an expression for the seller's expected revenue given any Bayesian incentive compatible mechanism. Expected revenue to the seller

$$
=\sum_{i=1}^{n} E_{\theta}\left[-t_{i}(\theta)\right] .
$$

Now, we have:

$$
E_{\theta}\left[-t_{i}(\theta)\right]=E_{\theta_{i}}\left[-E_{\theta_{-i}}\left[t_{i}(\theta)\right]\right]
$$




$$
\begin{aligned}
& =\int_{\underline{\theta_{i}}}^{\overline{\theta_{i}}}\left[\overline{y_{i}}\left(\theta_{i}\right) \theta_{i}-U_{i}\left(\theta_{i}\right)\right] \phi_{i}\left(\theta_{i}\right) d \theta_{i} \\
& =\int_{\underline{\theta_{i}}}^{\overline{\theta_{i}}}\left[\left[\overline{y_{i}}\left(\theta_{i}\right) \theta_{i}-U_{i}\left(\underline{\theta_{i}}\right)\right]-\int_{\underline{\theta_{i}}}^{\theta_{i}} \overline{y_{i}}(s) d s\right] \phi_{i}\left(\theta_{i}\right) d \theta_{i} .
\end{aligned}
$$

The last step is an implication of Myerson's characterization of Bayesian incentive compatible functions in linear environment. The above expression is now equal to

$$
=\left[\int_{\underline{\theta_{i}}}^{\overline{\theta_{i}}}\left(\overline{y_{i}}\left(\theta_{i}\right) \theta_{i}-\int_{\underline{\theta_{i}}}^{\theta_{i}} \overline{y_{i}}(s) d s\right) \phi_{i}\left(\theta_{i}\right) d \theta_{i}\right]-U_{i}\left(\underline{\theta_{i}}\right) .
$$

Now, applying integration by parts with $\int_{\underline{\theta_{i}}}^{\theta_{i}} \overline{y_{i}}(s) d s$ as the first function, we get

$$
\begin{aligned}
& \int_{\underline{\theta_{i}}}^{\overline{\theta_{i}}}\left(\int_{\underline{\theta_{i}}}^{\theta_{i}} \overline{y_{i}}(s) d s\right) \phi_{i}\left(\theta_{i}\right) d \theta_{i} \\
= & \int_{\theta_{\theta_{i}}}^{\overline{\theta_{i}}} \overline{y_{i}}\left(\theta_{i}\right) d \theta_{i}-\int_{\underline{\theta_{i}}}^{\overline{\theta_{i}}} \overline{y_{i}}\left(\theta_{i}\right) \Phi_{i}\left(\theta_{i}\right) d \theta_{i} \\
= & \int_{\underline{\theta_{i}}}^{\overline{\theta_{i}}} \overline{y_{i}}\left(\theta_{i}\right)\left[1-\Phi_{i}\left(\theta_{i}\right)\right] d \theta_{i} .
\end{aligned}
$$

Therefore we get

$$
\begin{aligned}
E_{\theta_{i}}\left[-\overline{t_{i}}\left(\theta_{i}\right)\right]= & -U_{i}\left(\underline{\theta_{i}}\right)+\left[\int_{\underline{\theta_{i}}}^{\overline{\theta_{i}}} \overline{y_{i}}\left(\theta_{i}\right)\left\{\theta_{i}-\frac{1-\Phi_{i}\left(\theta_{i}\right)}{\phi_{i}\left(\theta_{i}\right)}\right\} \phi_{i}\left(\theta_{i}\right) d \theta_{i}\right] \\
= & -U_{i}\left(\underline{\theta_{i}}\right)+\left[\int_{\underline{\theta_{1}}}^{\overline{\theta_{1}}} \cdots \int_{\underline{\theta_{n}}}^{\overline{\theta_{i}}} y_{i}\left(\theta_{1}, \cdots, \theta_{n}\right)\right. \\
& \left.\times\left(\theta_{i}-\frac{1-\Phi_{i}\left(\theta_{i}\right)}{\phi_{i}\left(\theta_{i}\right)}\right)\left(\prod_{j=1}^{n} \phi_{j}\left(\theta_{j}\right)\right) d \theta_{n} \cdots d \theta_{1}\right]
\end{aligned}
$$

since

$$
\overline{y_{i}}\left(\theta_{i}\right)=\int_{\underline{\theta_{1}}}^{\overline{\theta_{1}}} \cdots \int_{\underline{\theta_{n}}}^{\overline{\theta_{n}}} y_{i}\left(\theta_{1}, \cdots, \theta_{n}\right) \underbrace{d \theta_{n} \cdots d \theta_{1}}_{\text {without } d \theta_{i}} .
$$

Therefore the expected revenue of the seller

$$
\begin{aligned}
= & {\left[\int_{\underline{\theta_{1}}}^{\overline{\theta_{1}}} \cdots \int_{\underline{\theta_{n}}}^{\overline{\theta_{n}}} \sum_{i=1}^{n} y_{i}\left(\theta_{1}, \cdots, \theta_{n}\right)\left(\theta_{i}-\frac{1-\Phi_{i}\left(\theta_{i}\right)}{\phi_{i}\left(\theta_{i}\right)}\right)\right]\left(\prod_{j=1}^{n} \phi_{j}\left(\theta_{j}\right)\right) d \theta_{n} \cdots d \theta_{1} } \\
& -\sum_{i=1}^{n} U_{i}\left(\underline{\theta_{i}}\right) .
\end{aligned}
$$

By looking at the above expression, we see that any two Bayesian incentive compatible social choice functions that generate the same functions $\left(y_{1}(\theta), \cdots, y_{n}(\theta)\right)$ and the same values of $\left(U_{1}\left(\underline{\theta_{1}}\right), \cdots, U_{n}\left(\underline{\theta_{n}}\right)\right)$ generate the same expected revenue to the seller. 
As an application of the above theorem, we now state and prove a revenue equivalence theorem for a single indivisible item auction. The article by McAfee and McMillan [1] is an excellent reference for this topic and a part of the discussion in this section is inspired by this article.

\subsection{Revenue Equivalence of Four Classical Auctions}

There are four basic types of auctions when a single indivisible item is to be sold:

1. English auction: This is also called oral auction, open auction, open cry auction, and ascending bid auction. Here, the price starts at a low level and is successively raised until only one bidder remains in the fray. This can be done in several ways: (a) an auctioneer announces prices, (b) bidders call the bids themselves, or (b) bids are submitted electronically. At any point of time, each bidder knows the level of the current best bid. The winning bidder pays the latest going price.

2. Dutch auction: This is also called a descending bid auction. Here, the auctioneer announces an initial (high) price and then keeps lowering the price iteratively until one of the bidders accepts the current price. The winner pays the current price.

3. First price sealed bid auction: Recall that in this auction, potential buyers submit sealed bids and the highest bidder is awarded the item. The winning bidder pays the price that he has bid.

4. Second price sealed bid auction: This is the classic Vickrey auction. Recall that potential buyers submit sealed bids and the highest bidder is awarded the item. The winning bidder pays a price equal to the second highest bid (which is also the highest losing bid).

When a single indivisible item is to be bought or procured, the above four types of auctions can be used in a reverse way. These are called reverse auctions or procurement auctions. In this section, we would be discussing the Revenue Equivalence Theorem as it applies to selling. The procurement version can be analyzed on similar lines.

\subsubsection{The Benchmark Model}

There are four assumptions underlying the derivation of the Revenue Equivalence Theorem: (1) risk neutrality of bidders; (2) bidders have independent private values; (3) bidders are symmetric; (4) payments depend on bids alone. These are described below in more detail.

\section{(1) Risk Neutrality of Bidders}

It is assumed in the benchmark model that all the bidders are risk neutral. This immediately implies that the utility functions are linear.

\section{(2) Independent Private Values Model}

In the independent private values model, each bidder knows precisely how much he values the item. He has no doubt about the true value of the item to him. However, each bidder does not know anyone else's valuation of the item. Instead, he perceives any other bidder's valuation as a draw from some known probability distribution. Also, each bidder knows that the other bidders and the 
seller regard his own valuation as being drawn from some probability distribution. More formally, let $N=\{1,2, \cdots, n\}$ be the set of bidders. There is a probability distribution $\Phi_{i}$ from which bidder $i$ draws his valuation $v_{i}$. Only bidder $i$ observes his own valuation $v_{i}$, but all other bidders and the seller know the distribution $\Phi_{i}$. Any one bidder's valuation is statistically independent from any other bidder's valuation.

An apt example of this assumption is provided by the auction of an antique in which the bidders are consumers buying for their own use and not for resale. Another example is government contract bidding when each bidder knows his own production cost if he wins the contract.

A contrasting model is the common value model. Here, if $V$ is the unobserved true value of the item, then the bidders' perceived values $v_{i}, i=1,2, \cdots, n$ are independent draws from some probability distribution $H\left(v_{i} \mid V\right)$. All the bidders know the distribution $H$. An example is provided by the sale of an antique that is being bid for by dealers who intend to resell it. The item has one single objective value, namely its market price. However, no one knows the true value. The bidders, perhaps having access to different information, have different guesses about how much the item is objectively worth. Another example is that of the sale of mineral rights to a particular tract of land. The objective value here is the amount of mineral actually lying beneath the ground. However no one knows its true value.

Suppose a bidder were somehow to learn another bidder's valuation. If the situation is described by the common value model, then the above provides useful information about the likely true value of the item, and the bidder would probably change his own valuation in light of this. If the situation is described by the independent private values model, the bidder knows his own mind, and learning about others' valuations will not cause him to change his own valuation (although he may, for strategic reasons, change his bid).

Real world auction situations are likely to contain aspects of both the independent private values model and the common value model. It is assumed in the benchmark model that the independent private values assumption holds.

\section{(3) Symmetry}

This assumption implies that all the bidders have the same set of possible valuations, and further they draw their valuations using the same probability density $\phi$. That is, $\phi_{1}=\phi_{2}=\ldots=\phi_{n}=\phi$.

\section{(4) Dependence of Payments on Bids Alone}

It is assumed that the payment to be made by the winner to the auctioneer is a function of bids alone.

Theorem 1.2 (Revenue Equivalence Theorem for Single Indivisible Item Auctions) Consider a seller or an auctioneer trying to sell a single indivisible item in which $n$ bidders are interested. For the benchmark model (bidders are risk neutral, bidders have independent private values, bidders are symmetric, and payments depend only on bids), all the four basic auction types (English auction, Dutch auction, first price auction, and second price auction) yield the same average revenue to the seller.

The result looks counter intuitive: For example, it might seem that receiving the highest bid in a first price sealed bid auction must be better for the seller than receiving the second highest bid, as in second price auction. However, it is to be noted that bidders act differently in different auction situations. In particular, they bid more aggressively in a second price auction than in a first price auction. 
Proof: The proof proceeds in three parts. In Part 1, we show that the first price auction and the second price auction yield the same expected revenue in their respective equilibria. In Part 2, we show that the Dutch auction and the first price auction produce the same outcome. In Part 3, we show that the English auction and the second price auction yield the same outcome.

\section{Part 1: Revenue Equivalence of First Price Auction and Second Price Auction}

The first price auction and the second price auction satisfy the conditions of the theorem on revenue equivalence of two auctions.

- In both the auctions, the bidder with the highest valuation wins the auction.

- bidders' valuations are drawn from $\left[\underline{\theta_{i}}, \overline{\theta_{i}}\right]$ and a bidder with valuation at the lower limit of the interval has a payoff of zero in both the auctions.

Thus the theorem can be applied to the equilibria of the two auctions: Note that in the case of the first price auction, it is a Bayesian Nash equilibrium while in the case of the second price auction, it is a weakly dominant strategy equilibrium. In fact, it can be shown in any symmetric auction setting (where the bidders' valuations are independently drawn from identical distributions) that the conditions of the above proposition will be satisfied by any Bayesian Nash equilibrium of the first price auction and the weakly dominant strategy equilibrium of the second price scaled bid auction.

\section{Part 2: Revenue Equivalence of Dutch Auction and First Price Auction}

To see this, consider the situation facing a bidder in these two auctions. In each case, the bidder must choose how high to bid without knowing the other bidders' decisions. If he wins, the price he pays equals his own bid. This result is true irrespective of which of the assumptions in the benchmark model apply. Note that the equilibrium in the underlying Bayesian game in the two cases here is a Bayesian Nash equilibrium.

\section{Part 3: Revenue Equivalence of English Auction and Second Price Auction}

First we analyze the English auction. Note that a bidder drops out as soon the going price exceeds his valuation. The second last bidder drops out as soon as the price exceeds his own valuation. This leaves only one bidder in the fray and he wins the auction. Note that the winning bidder's valuation is the highest among all the bidders and he earns some payoff in spite of the monopoly power of the seller. Only the winning bidder knows how much payoff he receives because only he knows his own valuation. Suppose the valuations of the $n$ bidders are $v_{(1)}, v_{(2)}, \cdots, v_{(n)}$. Since the bidders are symmetric, these valuations are draws from the same distribution and without loss of generality, assume that these are in descending order. The winning bidder gets a payoff of $v_{(1)}-v_{(2)}$.

Next we analyze the second price auction. In the second price auction, the bidder's choice of bid determines only whether or not he wins; the amount he pays if he wins is beyond his control. We have already shown that each bidder's equilibrium best response strategy is to bid his own valuation for the item. The payment here is equal to the actual valuation of the bidder with the second highest valuation. Thus the expected payment and payoff are the same in English auction and the second price auction. This establishes Part 3 and therefore proves the Revenue Equivalence Theorem.

Note that the outcomes of the English auction and the second price auction satisfy a weakly dominant strategy equilibrium. That is, each bidder has a well defined best response bid regardless 
of how high he believes his rivals will bid. In the second price auction, the weakly dominant strategy is to bid true valuation. In the English auction, the weakly dominant strategy is to remain in the bidding process until the price reaches the bidder's own valuation.

\subsubsection{Some Observations}

We now make a few important observations.

- The theorem does not imply that the outcomes of the four auction forms are always exactly the same. They are only equal on average.

- Note that in the English auction or the second price auction, the price exactly equals the valuation of the bidder with the second highest valuation, $v_{(2)}$. In Dutch auction or the first price auction, the price is the expectation of the second highest valuation conditional on the winning bidder's own valuation. The above two prices will be equal only by accident; however, they are equal on average.

- Bidding strategy is very simple in the English auction and the second price auction. In the former, a bidder remains in bidding until the price reaches his valuation. In the latter, he submits a sealed bid equal to his own valuation.

- On the other hand, the bidding logic is quite complex in the Dutch auction and the first price auction. Here the bidder bids some amount less than his true valuation. Exactly how much less depends upon the probability distribution of the other bidders' valuations and the number of competing bidders. Finding the Nash equilibrium bid is a non-trivial computational problem.

- The Revenue Equivalence Theorem for the single indivisible item is devoid of empirical predictions about which type of auction will be chosen by the seller in any particular set of circumstances. However when the assumptions of the benchmark model are relaxed, particular auction forms emerge as being superior.

- The variance of revenue is lower in English auction or second price auction than in Dutch auction first price auction. Hence if the seller were risk averse, he would choose English or second price rather than Dutch or first price.

For more details on the revenue equivalence theorems, the reader is referred to the papers by Myerson [2], McAfee and McMillan [1], Klemperer [3], and the books by Milgrom [4] and Krishna [5].

\section{References}

[1] R. P. McAfee and J. McMillan. Auctions and bidding. Journal of Economic Literature, 25(2):699$738,1987$.

[2] Roger B. Myerson. Optimal auction design. Mathematics of Operations Research, 6(1):58-73, 1981.

[3] P. Klemperer. Why every economist should learn some auction theory. In M. Dewatripont, L. Hansen, and S. Turnovsky, editors, Advances in Economics and Econometrics: Invited Lectures to 8th World Congress of the Econometric Society. Cambridge University Press, Cambridge, UK, 2003. 
[4] Paul Milgrom. Putting Auction Theory to Work. Cambridge University Press, Cambridge, UK, 2004.

[5] Vijay Krishna. Auction Theory. Academic Press, San Diego, California, USA, 2002. 